\title{
Biofilm of Pseudomonas aeruginosa in Nosocomial Infection
}

\author{
Z. Mahmmudi ${ }^{1} \&$ A. A. Gorzin ${ }^{2}$ \\ ${ }^{1}$ Kazeroun Branch, Islamic Azad University, Kazeroon, Iran \\ ${ }^{2}$ School of Medicine, Shiraz University of Medical Sciences, Shiraz, Iran \\ Correspondence: A. A. Gorzin, School of Medicine, Shiraz University of Medical Sciences, Shiraz, Iran. E-mail: \\ z.mahmmudi.792@gmail.com
}

Received: December 27, 2016

Accepted: February 4, $2017 \quad$ Online Published: February 9, 2017

doi:10.5539/jmbr.v7n1p29

URL: http://dx.doi.org/10.5539/jmbr.v7n1p29

\begin{abstract}
Bacteria in natural, industrial and clinical settings predominantly live in biofilms, i.e., sessile structured microbial communities encased in self-produced extracellular matrix material. One of the most important characteristics of microbial biofilms is that the resident bacteria display a remarkable increased tolerance toward antimicrobial attack. Biofilms formed by opportunistic pathogenic bacteria are involved in devastating persistent medical deviceassociated infections, and chronic infections in individuals who are immune-compromised or otherwise impaired in the host defense. Because the use of conventional antimicrobial compounds in many cases cannot eradicate biofilms, there is an urgent need to develop alternative measures to combat biofilm infections. The present review is focussed on the important opportunistic pathogen and biofilm model organism Pseudomonas aeruginosa. Initially, biofilm infections where P. aeruginosa plays an important role are described. Subsequently, current insights into the molecular mechanisms involved in P. aeruginosa biofilm formation and the associated antimicrobial tolerance are reviewed. And finally, based on our knowledge about molecular biofilm biology, a number of therapeutic strategies for combat of $\mathrm{P}$. aeruginosa biofilm infections are presented.
\end{abstract}

Keywords: Pseudomonas aeruginosa; biofilm matrix; exopolysaccharides; gene regulation; anti-biofilm

\section{Introduction}

Biofilms are microbial communities encased in extracellular polymeric substances (EPS) (Epps \& Walker, 2006). Biofilm formation represents a protective mode of growth that allows microorganisms to survive in hostile environments and disperse seeding cells to colonize new niches under desirable conditions. Biofilms can form on a variety of surfaces and are prevalent in natural, industrial, and hospital niches. These sessile OPEN ACCESS Int. J. Mol. Sci. 2013, 1420984 microbial communities are physiologically distinct from free-living planktonic counterparts (English \& Gaur, 2010; Guarner \& Malagelada, 2003). Clinically, biofilms are responsible for many persistent and chronic infections due to their inherent resistance to antimicrobial agents and the selection for phenotypic variants. A better understanding of the genetic and molecular mechanisms of biofilm formation may provide strategies for the control of chronic infections and problems related to biofilm formation. The EPS of biofilm is a mixture of polysaccharides, extracellular DNA (eDNA), and proteins, which function as matrix, or glue, holding microbial cells together. The biofilm matrix contributes to the overall architecture and the resistance phenotype of biofilms (Beaugerie \& Petit, 2004; Høiby, Ciofu, \& Bjarnsholt, 2010). Uncovering roles played by EPS matrices in biofilm formation will be beneficial for the design of targeted molecules to control biofilm formation. In this review, advances in biofilm formation and regulation are presented with a focus on the biofilm matrix in P. aeruginosa, a model organism for biofilm research.

\section{The Mechanism of Biofilm Formation}

Formation of a biofilm begins with the attachment of free-floating microorganisms to a surface. While still not fully understood, it is thought that the first colonists of a biofilm adhere to the surface initially through weak, reversible adhesion via van der Waals forces and hydrophobic effects (Kalia \& Purohit, 2011; Blackledge, Worthington, \& Melander, 2013). If the colonists are not immediately separated from the surface, they can anchor themselves more permanently using cell adhesion structures such as pili. Hydrophobicity also plays an important role in determining the ability of bacteria to form biofilms, as those with increased hydrophobicity have reduced repulsion between the extracellular matrix and the bacterium (Sharma et al., 2014). 
Some species are not able to attach to a surface on their own but are instead able to anchor themselves to the matrix or directly to earlier colonists. It is during this colonization that the cells are able to communicate via quorum sensing (QS) using products such as N-acyl homoserine lactone (AHL). Some bacteria are unable to form biofilms as successfully due to their limited motility. Non-motile bacteria cannot recognize the surface or aggregate together as easily as motile bacteria (Sharma et al., 2014). Once colonization has begun, the biofilm grows through a combination of cell division and recruitment. Polysaccharide matrices typically enclose bacterial biofilms. In addition to the polysaccharides, these matrices may also contain material from the surrounding environment, including but not limited to minerals, soil particles, and blood components, such as erythrocytes and fibrin (Sharma et al., 2014). The final stage of biofilm formation is known as dispersion, and is the stage in which the biofilm is established and may only change in shape and size.

The development of a biofilm may allow for an aggregate cell colony (or colonies) to be increasingly resistant to antibiotics. Cell-cell communication or quorum sensing has been shown to be involved in the formation of biofilm in several bacterial species (Deep, Chaudhary, \& Gupta, 2011).

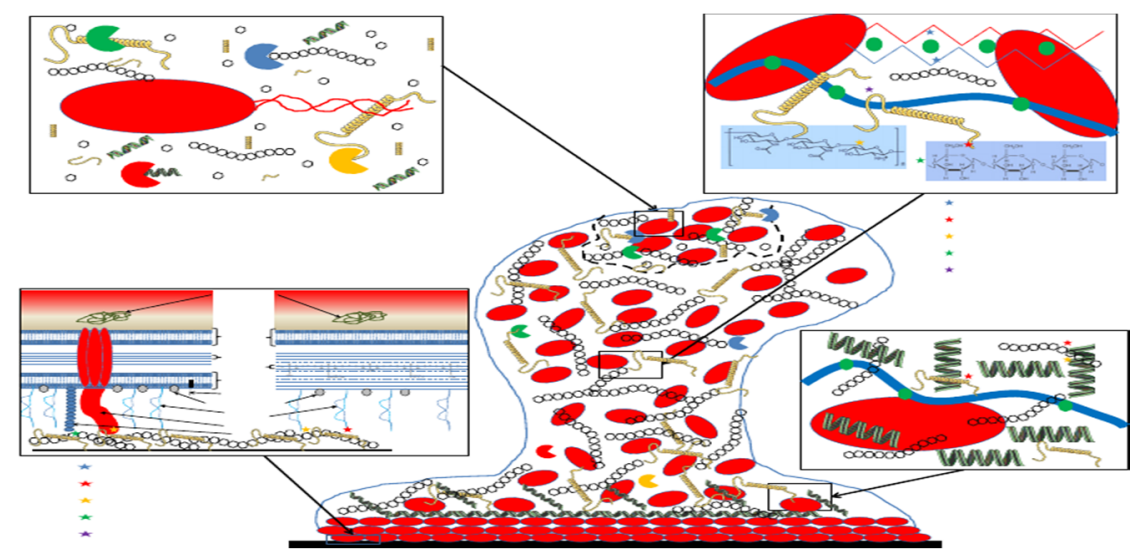

Figure 1. The biofilm matrix is comprised of entangled polymers (polysaccharides, DNA, proteins) that affect the permeability and mechanical properties of the entire biofilm. To understand the biophysical properties of the biofilm several questions need to be addressed. For example, what is the pore size of the matrix? Does a specific substrate interact with the matrix components? Which structural components of the matrix regulate the permeability properties? Is the matrix a static arrangement or do the individual components engage in dynamic rearrangements?

\subsection{Biofilm associated infections and their implications in nosocomial infection}

According to a recent public announcement from the National Institutes of Health, more than $60 \%$ of all the infections are caused by biofilms (Karatan \& Watnick, 2009). As described by Prasanna et al, about $40-50 \%$ of adults had biofilm related gingival infections. Among 4000 infants with cerebrospinal- fluid shunts, $15-20 \%$ had biofilm related infections. $95 \%$ of the urinary tract infections were associated with urinary catheters. $86 \%$ pneumonias were associated with mechanical ventilation and $85 \%$ of the blood stream infections were closely related to intravascular devices (Guarner \& Malagelada, 2003).

\subsection{The Detection of Biofilm Producing Microorganisms}

Early biofilm formation detection might result in a greater success in the treatment, because in long standing cases, they may be very damaging and may produce immune complex sequelae (Yang et al., 2012). There are two methods for the detection of biofilms - 1). The Phenotypic method a. The tissue culture plate (TCP) method - The wells of the tissue culture plates are inoculated with a bacterial suspension along with positive and negative controls and these are incubated for 24 to 48 hours. Planktonic cells are removed by washing with phosphate buffered saline. Biofilms are fixed with $2 \%$ sodium acetate and are stained with $0.1 \%$ crystal violet. The excess dye is washed away with deionised water. The plates are dried properly and the optical densities of the stained biofilms are obtained spectrophotometrically. $b$. The tube method(TM) $-10 \mathrm{ml}$ of Tripticase soy broth with $1 \%$ glucose is inoculated with a loopful of test organisms, along with positive and negative controls. The broths are incubated at for $24-48$ hours. The culture supernatants are decanted and the tubes are washed with phosphate buffered saline. The tubes are dried and are stained with $0.1 \%$ crystal violet. The excess stain is washed away with deionised water. The tubes are dried in an inverted position. c. The Congo red agar (CRA) method - The Congo 
red stain is prepared as a concentrated aqueous solution and is autoclaved at $1210 \mathrm{c}$ for 15 minutes. This is added to autoclaved Brain heart infusion agar with sucrose at 550c. The plates are inoculated with the test organisms along with positive and negative controls and are incubated at 370C for 24 to 48 hours aerobically. Black colonies with a dry crystalline consistency indicate biofilm production. Various studies have established that TCP is a better screening test for biofilm production than the TM and the CRA methods. The test is easy to perform and to assess biofilms, both qualitatively and quantitatively (Nikolaev \& Plakunov, 2007; Flemming, Neu, \& Wozniak, 2007). 2). The Genotypic method Sonications and PCR amplification methods have been shown to improve the detection of biofilms. Biofilm non producers are negative for ica A and ica D and lack the entire ica ADBC operon. But this requires specialized equipments and techniques (Sutherland, 2001; Branda, Vik, Friedman, \& Kolter, 2005).

\subsection{Regulation of Biofilm Matrix in P. aeruginosa}

Gene regulation is important for our understanding of biofilm formation. Generally, organisms form a biofilm in response to several factors including nutritional cues, secondary messengers, host-derived signals or, in some cases, to sub-inhibitory concentrations of antibiotics (Hentzer, Eberl, \& Givskov, 2005; Shrout et al., 2006). When a cell switches to the biofilm mode of growth, it undergoes a phenotypic shift in behavior whereby a large array of genes is differentially regulated (Ma et al., 2009). Biofilm formation is a multicellular process involving environmental signals and a concerted regulation combining both environmental signals and regulatory networks. Due to the major roles of EPS matrix in biofilm formation, its regulation is discussed. Int. J. Mol. Sci. 2013, 1420990 3.1. cdi-GMP Bis-(3'-5')-cyclic dimeric guanosine monophosphate (c-di-GMP), a ubiquitous intracellular second messenger widely distributed in bacteria, was discovered in 1987 as an allosteric activator of the cellulose synthase complex in Gluconacetobacter xylinus (Shrout et al., 2006). In general, c-di-GMP stimulates the biosynthesis of adhesins and exopolysaccharide mediated biofilm formation and inhibits bacterial motilities, which controls the switch between the motile planktonic and sessile biofilm-associated lifestyle of bacteria (Figure 2). Moreover, cdi-GMP controls the virulence of animal and plant pathogens, progression through the cell cycle, antibiotic production and other cellular functions (Branda, Vik, Friedman, \& Kolter, 2005; Ryder, Byrd, \& Wozniak, 2007).

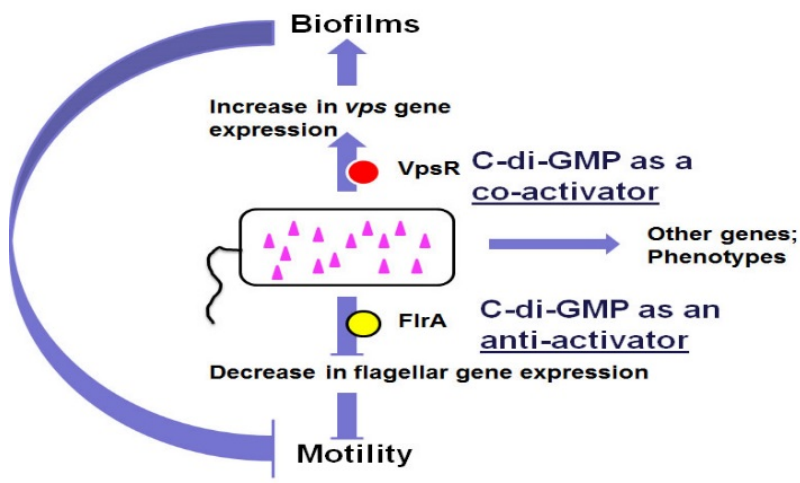

Figure 2. Schematic presentation of physiological functions of c-di-GMP. In bacterial cells, c-di-GMP is generated by diguanylate cyclases (DGC) and broken down by specific phosphodiesterases (PDE). As a second messenger, low levels of c-di-GMP can promote motility by upregulating flagellar expression, assembly or interfering with flagellar motor function and are required for the expression of acute virulence genes. High levels of c-di-GMP however favor sessility and stimulate the synthesis of various matrix exopolysaccharides, such as Pel (mediated by PelD) and alginate (mediated by Alg44) (Masák, Čejková, Schreiberová, \& Řezanka, 2014; Donlan, 2002; Karatan \& Watnick, 2009)

\subsection{Matrix-Driven Strategies against Biofilms}

Once biofilms develop into a mature stage, they become extremely difficult to eradicate from infections sites with traditional antimicrobial agents (Cegelski, Marshall, Eldridge, \& Hultgren, 2008). Agents that inhibit biofilm formation or transform bacteria from biofilm life style to free-living individuals are ideal to eradicate biofilm. The strategies used for anti-biofilm mainly stem from two basic ways: matrix synthesis and its regulatory mechanisms. For example, disruption of the initial attachment that is dependent on a large array of adhesins would contribute to inhibition of the establishment of biofilms, while the digestion of the EPS matrix may be another method to interfere with biofilm formation. As we mentioned before, DNase I treatment has already shown efficacy in the inhibition of the early development of biofilm. It was also reported that alginate lyase could enhance antibiotic 
killing of mucoid P. aeruginosa in biofilms (Bjarnsholt, 2013). In addition, the macrolide antibiotic azithromycin was shown to block alginate formation and quorum sensing signaling (Nikolaev \& Plakunov, 2007) and was further reported to improve lung function of CF patients, especially in the subgroup chronically colonized by Pseudomonas (Flemming, Neu, \& Wozniak, 2007). Antagonizing the intracellular signaling molecules to control biofilm formation has also been investigated. One example is the identification of furanones, which have shown their ability to inhibit Int. J. Mol. Sci. 2013, 1420996 the biofilm formation of P. aeruginosa in vitro (Sutherland, 2001; Branda, Vik, Friedman, \& Kolter, 2005). Molecules of this type have been reported to function through inhibiting the AHL-dependent QS systems in P. aeruginosa. Iron has also been employed in distinct aspects to control the formation of biofilms. Singh and his colleagues have identified an innate immunity component, lactoferrin, which prevents P. aeruginosa biofilm formation by chelating iron and stimulating the type IV pilimediated twitching motility (Epps \& Walker, 2006; Yang et al., 2012). Furthermore, iron salts such as ferric ammonium citrate were found to not only perturb biofilm formation but also disrupt existing biofilms by P. aeruginosa (Shrout et al., 2006). In a screen of co-therapy of antibiotics against P. aeruginosa, 14-alpha-lipoyl and rographolide, a diterpenoid lactone derivative from the herb Andrographis paniculata appeared to inhibit biofilm formation by decreasing EPS production and to sensitize the bacterium to a variety of antibiotics (Yang et al., 2012). Recently, it was found that Gram-positive bacterium Bacillus subtilis produced a factor that prevented biofilm formation and could break down existing biofilms. The factor was identified to be a mixture of D-leucine, D-methionine, D-tyrosine, and D-tryptophan that could disassemble at nanomolar concentrations. D-amino acid treatment subserved the release of amyloid fibers that linked cells together in the biofilm. In addition, D-amino acids also prevented biofilm formation by Staphylococcus aureus and P. aeruginosa, indicating it may be a widespread signal for biofilm disassembly (Chicurel, 2000). Furthermore, the same group identified another biofilm disassembly compound, norspermidine, which targets directly and specifically with the exopolysaccharide matrix and this biofilm inhibition effect could be enhanced together with D-amino acids and is effective in other bacterial species (Masák, Čejková, Schreiberová, \& Řezanka, 2014).

\subsection{Perspectives}

Accumulating data presented in the recent literature provides valuable insights into the novel roles of the biofilm matrix and its regulatory mechanism in P. aeruginosa biofilm formation. A deep understanding of the mechanisms involved in biofilm formation will ultimately shed light on the generation of alternative treatments for P. aeruginosa infections. There is no doubt that future studies will reveal additional biofilm matrix components and identify more elaborate regulatory circuits for biofilm formation. Finally, the interaction of the biofilm matrix and the synergistic effects of different anti-biofilm strategies should also be regarded as major concerns.

\section{Conclusion}

Many biofilm infections develop slowly, producing very few symptoms initially, but in the long run, they may produce immune complex sequelae and may act as reservoirs of infection (English \& Gaur, 2010). Standard, in vitro antibiotic susceptibility tests are not predictive of the therapeutic outcome of biofilm associated infections (Ghafoor, Hay, \& Rehm, 2011). The overall healthcare costs which are attributed to the treatment of biofilm associated infections are much higher due to their persistence. Besides, a longer hospital stay is another factor for higher costs. Early detection of biofilm associated infections and newer treatment options for the management of the same are needed.

\section{References}

Beaugerie, L., \& Petit, J. C. (2004). Antibiotic-associated diarrhoea. Best practice \& research Clinical gastroenterology, 18(2), 337-352.

Bjarnsholt, T. (2013). The role of bacterial biofilms in chronic infections. Apmis, 121(s136), 1-58.

Blackledge, M. S., Worthington, R. J., \& Melander, C. (2013). Biologically inspired strategies for combating bacterial biofilms. Current opinion in pharmacology, 13(5), 699-706.

Branda, S. S., Vik, Å., Friedman, L., \& Kolter, R. (2005). Biofilms: the matrix revisited. Trends in microbiology, 13(1), 20-26.

Cegelski, L., Marshall, G. R., Eldridge, G. R., \& Hultgren, S. J. (2008). The biology and future prospects of antivirulence therapies. Nature Reviews Microbiology, 6(1), 17-27.

Chicurel, M. (2000). Bacterial biofilms and infections. Slimebusters. Nature, 408(6810), 284-286.

Costerton, J. W., Stewart, P. S., \& Greenberg, E. P. (1999). Bacterial biofilms: a common cause of persistent infections. Science, 284(5418), 1318-1322. 
Davey, M. E., Caiazza, N. C., \& O'Toole, G. A. (2003). Rhamnolipid surfactant production affects biofilm architecture in Pseudomonas aeruginosa PAO1. Journal of bacteriology, 185(3), 1027-1036.

Deep, A., Chaudhary, U., \& Gupta, V. (2011). Quorum sensing and bacterial pathogenicity: from molecules to disease. Journal of laboratory physicians, 3(1), 4-11.

Donlan, R. M. (2002). Biofilms: microbial life on surfaces. Emerg Infect Dis, 8(9), 881-890.

English, B. K., \& Gaur, A. H. (2010). The use and abuse of antibiotics and the development of antibiotic resistance. In Hot Topics in Infection and Immunity in Children VI (pp. 73-82). Springer New York.

Epps, L. C., \& Walker, P. D. (2006). Fluoroquinolone consumption and emerging resistance. US Pharm, 10, 47-54.

Flemming, H. C., \& Wingender, J. (2010). The biofilm matrix. Nature Reviews Microbiology, 8(9), 623-633.

Flemming, H. C., Neu, T. R., \& Wozniak, D. J. (2007). The EPS matrix: the "house of biofilm cells". Journal of bacteriology, 189(22), 7945-7947.

Ghafoor, A., Hay, I. D., \& Rehm, B. H. (2011). Role of exopolysaccharides in Pseudomonas aeruginosa biofilm formation and architecture. Applied and environmental microbiology, 77(15), 5238-5246.

Guarner, F., \& Malagelada, J. R. (2003). Gut flora in health and disease. The Lancet, 361(9356), 512-519.

Hentzer, Á., Eberl, Á., \& Givskov, Á. (2005). Transcriptome analysis of Pseudomonas aeruginosa biofilm development: anaerobic respiration and iron limitation. Biofilms, 2(01), 37-61.

Høiby, N., Ciofu, O., \& Bjarnsholt, T. (2010). Pseudomonas aeruginosa biofilms in cystic fibrosis. Future microbiology, 5(11), 1663-1674.

Kalia, V. C., \& Purohit, H. J. (2011). Quenching the quorum sensing system: potential antibacterial drug targets. Critical reviews in microbiology, 37(2), 121-140.

Karatan, E., \& Watnick, P. (2009). Signals, regulatory networks, and materials that build and break bacterial biofilms. Microbiology and Molecular Biology Reviews, 73(2), 310-347.

Ma, L., Conover, M., Lu, H., Parsek, M. R., Bayles, K., \& Wozniak, D. J. (2009). Assembly and development of the Pseudomonas aeruginosa biofilm matrix. PLoS Pathog, 5(3), e1000354.

Ma, L., Wang, J., Wang, S., Anderson, E. M., Lam, J. S., Parsek, M. R., \& Wozniak, D. J. (2012). Synthesis of multiple Pseudomonas aeruginosa biofilm matrix exopolysaccharides is post-transcriptionally regulated. Environmental microbiology, 14(8), 1995-2005.

Masák, J., Čejková, A., Schreiberová, O., \& Řezanka, T. (2014). Pseudomonas biofilms: possibilities of their control. FEMS microbiology ecology, 89(1), 1-14.

Nikolaev, Y. A., \& Plakunov, V. K. (2007). Biofilm—“City of microbes" or an analogue of multicellular organisms?. Microbiology, 76(2), 149-163.

Ryder, C., Byrd, M., \& Wozniak, D. J. (2007). Role of polysaccharides in Pseudomonas aeruginosa biofilm development. Current opinion in microbiology, 10(6), 644-648.

Sharma, G., Rao, S., Bansal, A., Dang, S., Gupta, S., \& Gabrani, R. (2014). Pseudomonas aeruginosa biofilm: potential therapeutic targets. Biologicals, 42(1), 1-7.

Shrout, J. D., Chopp, D. L., Just, C. L., Hentzer, M., Givskov, M., \& Parsek, M. R. (2006). The impact of quorum sensing and swarming motility on Pseudomonas aeruginosa biofilm formation is nutritionally conditional. Molecular microbiology, 62(5), 1264-1277.

Sutherland, I. W. (2001). The biofilm matrix-an immobilized but dynamic microbial environment. Trends in microbiology, 9(5), 222-227.

Watnick, P., \& Kolter, R. (2000). Biofilm, city of microbes. Journal of bacteriology, 182(10), 2675-2679.

Yang, L., Liu, Y., Wu, H., Song, Z., Høiby, N., Molin, S., \& Givskov, M. (2012). Combating biofilms. FEMS Immunology \& Medical Microbiology, 65(2), 146-157.

\section{Copyrights}

Copyright for this article is retained by the author(s), with first publication rights granted to the journal.

This is an open-access article distributed under the terms and conditions of the Creative Commons Attribution license (http://creativecommons.org/licenses/by/4.0/). 